\title{
Effect of Central Antileptin Antibody on the Onset of Female Rat Puberty
}

\author{
Ruimin Chen, ${ }^{1}$ Gail J. Mick, ${ }^{2,3}$ Rongxian Xu, ${ }^{4}$ Daoxin Zheng, ${ }^{1}$ Yanfeng Fan, ${ }^{4}$ \\ Xiangquan Lin, ${ }^{1}$ and Kenneth L. McCormick ${ }^{2}$ \\ ${ }^{1}$ Department of Endocrinology, Fuzhou Children's Hospital of Fujian, Fuzhou 350005, China \\ ${ }^{2}$ Division of Pediatric Endocrinology and Diabetes, University of Alabama at Birmingham, Birmingham, AL 35233, USA \\ ${ }^{3}$ Department of Pediatrics, University of Alabama at Birmingham, CPP 230, 1601 4th Avenue South, Birmingham, AL 35233, USA \\ ${ }^{4}$ Department of Nutrition and Maternal \& Children Care School of Public Health, Fujian Medical University, Fuzhou 350004, China
}

Correspondence should be addressed to Gail J. Mick, gmick@peds.uab.edu

Received 9 January 2009; Revised 4 July 2009; Accepted 29 July 2009

Recommended by David Zangen

\begin{abstract}
The effect of intracerebroventricular (ICV) antileptin antibody on the onset of puberty in the female rat and the relationship between serum leptin, luteinizing hormone (LH), and body weight were investigated. Antileptin antibody (group A) was infused ICV from days 23-36 in prepubertal female rats whereas the control (group B) received ICV goat immunoglobulin G (IgG). In the antileptin group, mean day of vaginal opening $(\mathrm{VO})$ was postponed (day 34 versus day $30, P<.01$ ). Body weight trended higher after 30 days in the antileptin group but not significantly. However, there was no difference in serum leptin and LH between the two groups on the day of VO. Serum leptin was relatively constant from day 23 through day 31 and did not correlate with LH $(r=0.14, P=.10)$. These studies demonstrate that central leptin promotes the onset of female rat puberty as evidenced by VO. Finally, central leptin impacts female rat pubertal onset in distinction from serum leptin and body weight.
\end{abstract}

Copyright (c) 2009 Ruimin Chen et al. This is an open access article distributed under the Creative Commons Attribution License, which permits unrestricted use, distribution, and reproduction in any medium, provided the original work is properly cited.

\section{Introduction}

It is well recognized that a requisite for the onset of human puberty is a healthy body composition, particularly a critical mass of body fat $[1,2]$. Low body fat prevents or hinders the onset of puberty $[3,4]$. For decades it has been proposed that a peripheral metabolic signal from adipose tissue oversees the onset of puberty. Leptin, an adipocyte hormone and product of the obese $(o b)$ gene, may be a primary candidate signal molecule among other complex metabolic signals [1].

In humans, leptin correlates most significantly with BMI and body fat [5]. Numerous studies have inferred that leptin may regulate the hypothalamic-pituitary-gonadal axis. One indication that leptin is involved in centrally regulated maturation of the reproductive system was the discovery that $o b / o b$ females are always sterile [6], and weight loss induced by dietary restriction fails to correct their sterility. Importantly, their fertility can be reversed by leptin treatment in both sexes $[7,8]$. The ob/ob leptin deficient and $\mathrm{db} / \mathrm{db}$ leptin resistant mouse models have greatly advance our understanding regarding the pivotal role of leptin in reproduction $[9,10]$. Moreover, the ability of leptin to accelerate the onset of puberty in normal female mice has been demonstrated [9].

In vitro and in vivo studies demonstrated an acute stimulatory effect of leptin on the hypothalamic-pituitary unit in adult rats [11]. Exogenous leptin produced a dose-related increase in follicle-stimulating hormone (FSH) and luteinizing hormone $(\mathrm{LH})$ release in healthy rats and isolated pituitary cells [11, 12]. Furthermore, leptin can increase the hypothalamic gonadotropin-releasing hormone $(\mathrm{GnRH})$ content at 15 days of age in female rats [13]. In agreement with this finding, studies in healthy mice demonstrated premature puberty after intraperitoneal leptin administration [14]. However, in another study concerning male rats, no correlation between simultaneous leptin and LH measurements was reported; the implication of this is that leptin does not modify puberty by direct actions on the hypothalamic-pituitary axis [15]. 
In short, the issue as to the role of leptin in the regulation of puberty is controversial. Also, conflicting data exists as to whether serum leptin concentrations increase temporarily during puberty in female. Moreover, the precise relationship between leptin and LH secretion in female rats through the prepubertal and peripubertal period has not been defined.

To address the issue, we infused antileptin antibody (experimental group A) versus IgG (control group B) in prepubertal female rats and monitored the time to puberty onset, as determined by vaginal opening $(\mathrm{VO})$ and sequential serum LH and leptin concentrations.

\section{Methods}

2.1. Animals. 21 Sprague-Dawley females rats, age 21 days and body weight $50 \pm 6 \mathrm{~g}$, were studied. All rats were born at the Shanghai Animals Institute. Rats were housed in a room at $23^{\circ}$ with a $12 / 12$ light/dark cycle. Starting on postnatal day 23, all animals were allowed free access to a standard laboratory rat chow and water. All of the following procedures were approved by the Fujian Medical University Animal Care and Use Committee (Fujian, China).

2.2. Immunoglobulins and Protein Assay. Protein was measured by a Coomassie Blue G-250 dye-binding assay. Goatderived antirat leptin and normal goat IgG were purchased from Pepro Tech (Rocky Hill, NJ, USA) and diluted to $0.1 \mu \mathrm{g} / \mu \mathrm{L}$ solution.

2.3. Intracerebroventricular Surgery. At 23 days of age, rats were randomly divided into antileptin antibody treated group A and control groups B, the latter receiving goat IgG. After pentobarbital anesthesia, all rats were implanted with a stainless steel cannula positioned stereotaxically into the left lateral ventricle of the brain via a hole drilled in the skull ( $1.3 \mathrm{~mm}$ lateral and $1.3 \mathrm{~mm}$ posterior to bregma), and the cannula was fixed at a depth of $4.0 \mathrm{~mm}$ from the skull surface.

Postoperatively, the health status of the rats was monitored several times daily.

2.4. Experimental Groups and Procedure. From day 23 to day 36 , group $\mathrm{A}(n=10)$ and $\mathrm{B}$ rats $(n=11)$ were injected ICV daily either with $6 \mathrm{uL}$ antileptin antibody (group A) or equivolume goat IgG (group B).

Body weights were measured every other day and VO was monitored two times per day at 9 AM and 9 PM. Blood samples were obtained at 9 AM via a tail vein on every other day from days 23 to 39.

The last blood sample was obtained on postnatal day 39. To confirm the cannula placement, on day 43 all rats were injected with a dye (Evans blue). Only those rats with the cannula positioned correctly in the lateral ventricle of the brain were included in the analysis. Before VO, one rat died during surgery in control, group B, and two rats in the antileptin, group A died. The two later rats (group A) appeared vigorous until death. No cause was identified.
2.5. Hormonal Assays. Leptin and LH blood samples were obtained at 9 AM every other day via tail vein. Serum was frozen at $-20^{\circ}$ until assayed for leptin and LH content.

2.6. Hormone and Metabolite Determinations. Serum was assayed for leptin using ELISA kit from Linco Research Inc. (St Charles, Mo, USA). Serum was assayed for LH using ELISA kits from Shanghai Lan Dun Inc. The sensitivities of the $\mathrm{LH}$ and leptin assays were $0.04 \mathrm{ng} / \mathrm{mL}$ and $0.01 \mathrm{ng} / \mathrm{mL}$, respectively. The inter- and intra-assay coefficients of variations were both less than 10\% for both assays.

2.7. Statistical Analysis. Results are expressed as the mean \pm SEM. T-tests (and nonparametric test) was used to analyze the data. The median day of VO is also given. Statistical correlations between serum leptin and LH were determined using the Pearson Product-Moment Correlation Coefficient. Differences were considered significant if $P$ was less than .05 .

\section{Results}

As illustrated in Figure 1, throughout the study the body weights in both groups were similar $(P>.05)$. While a slight deviation in weight gain was suggested after 35 days, these weight differences were not significant and, moreover, barely apparent between 30 and 34 days. Notably, the body weight on the day of $\mathrm{VO}$ in the antileptin antibody group was not significantly higher compared to the control group B (Figure 1). The rats that received antileptin antibody (group A) had a significant $(P<.01)$ delay in VO (mean \pm SEM VO group $\mathrm{A}=34 \pm 1$ days versus $30.2 \pm 0.7$ days in the control group B). The median VO in group A was 33 days (range 3039) versus a median VO of 30 days (range 27-34), control group B.

Figure 2 depicts serum leptin during sexual maturity after central antileptin antibody infusion. Serum concentrations of leptin were relatively constant from day 23 through day 31. Nevertheless, at day 35, day 37, and day 39 , there was a statistically significant increase in leptin concentrations in antileptin group A compared to the start day (day 23). This increase did not occur in the control group B. Unexplained also, a significant $(P<.0001)$ depression in serum leptin occurred on day 33 in antileptin group A.

The LH data, illustrated in Figure 3, revealed that both the antileptin treated group A and control group B had, when compared to the beginning of the experiment (day 23), significant increases in the serum $\mathrm{LH}$ concentrations from days 27 through 39 or from days 29 through 39, respectively. There was no significant difference in serum LH between the two groups on the same chronological day of age at any time point examined.

Finally, there was no significant correlation between serum leptin and LH $(r=0.14, P=.10)$ throughout the study period (data not shown). 


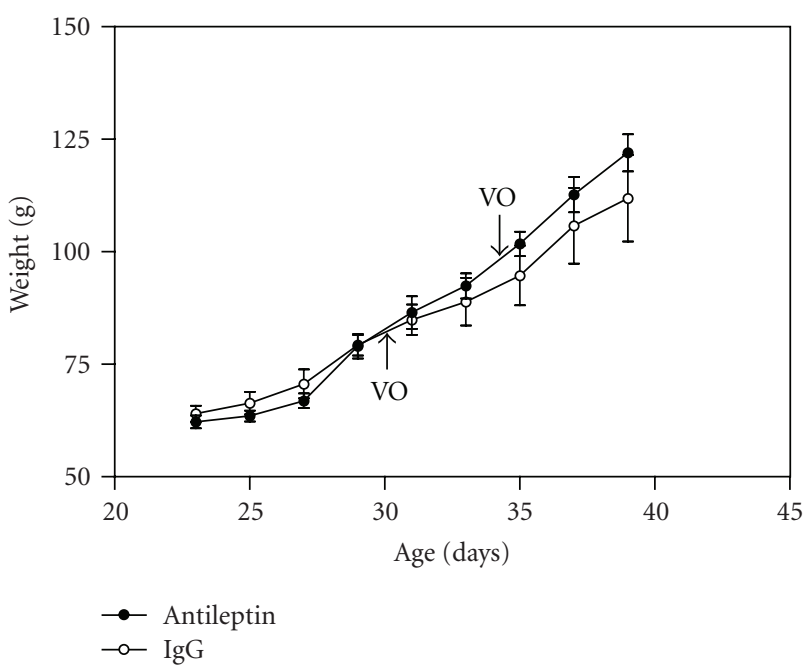

FIGURE 1: Body weight as rats approach and during puberty $(n=10$ in antileptin group $\mathrm{A}$, and $n=11$ in control group B). Data are expressed as mean \pm SEM. Arrows indicate the mean day of $\mathrm{VO}$ in each group (antileptin group $\mathrm{A}=34 \pm 1$ days versus $30.2 \pm 0.7$ days in the control group $\mathrm{B}(P<.01)$. Median VO data are given in the results. No difference in body weight $(P>.05)$ was observed between the two groups.

\section{Discussion}

By using normal healthy female rats, this study examined the effect of ICV antileptin antibody on the onset of rat puberty and circulating levels of leptin and LH. Remarkably, which one of the several biochemical trigger(s) or perhaps combinations thereof, governs the onset of puberty remains unproven $[10,16,17]$. The role of leptin in puberty is inferred in leptin receptor-deficient patients who have an associated hypogonadotropic hypogonadism $[18,19]$.

These adduced studies suggest that leptin may be a requisite metabolic signal necessary for the initiation of pubertal development. In the female rat, vaginal opening provides a reliable and conspicuous external sign for the onset of this process. In our studies, antileptin antibody administrated to group A was able to significantly postpone VO compared to control group B $(P<.01)$. Furthermore, as in the control group, serum leptin in group A was relatively constant from day 23 through day 31. A previous study likewise reported delayed VO in rats following ICV antileptin antibody, the extent of the delay depending on the feeding protocol of the rats [20].

Circulating leptin levels have been measured during pubertal development in several species. In female mice, a peak in plasma levels of leptin during the second week of postnatal life has been noted to occur independently of changes in body weight and prior to increases in estradiol. A significant elevation of plasma leptin during the peripubertal period was not observed [21]. Likewise, no change was detected in circulating daytime leptin in male rhesus macaques over the peripubertal period, during which time $\mathrm{LH}, \mathrm{FSH}$, and testosterone levels were approaching adult levels [22]. Similarly, female rats that underwent central

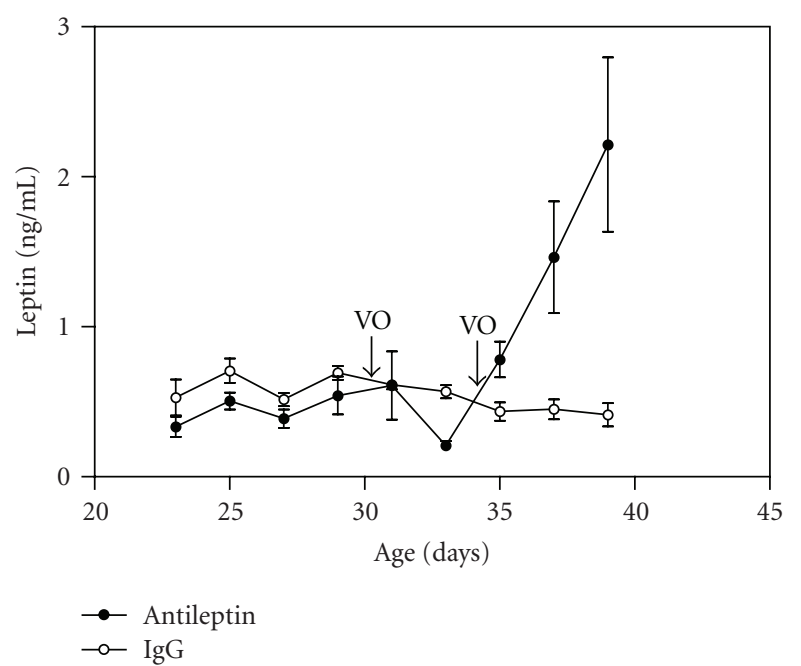

FIGURE 2: Serum leptin concentrations as rats approach and during puberty ( $n=10$ in antileptin group A and $n=11$ in control group B). Data are presented as mean \pm SEM. From day 23 through day 31 , no difference in leptin was observed between the two groups $(P>$ $.05)$. Nevertheless, at days 35,37 , and 39 , there was a statistically significant increase compared to day 23 in group A $(P=.013, .004$, .001 , resp.). For the control group of all ages, this rise in serum leptin did not occur $(P>.05)$. Arrows indicate the mean day of VO (see Figure 1 legend for details).

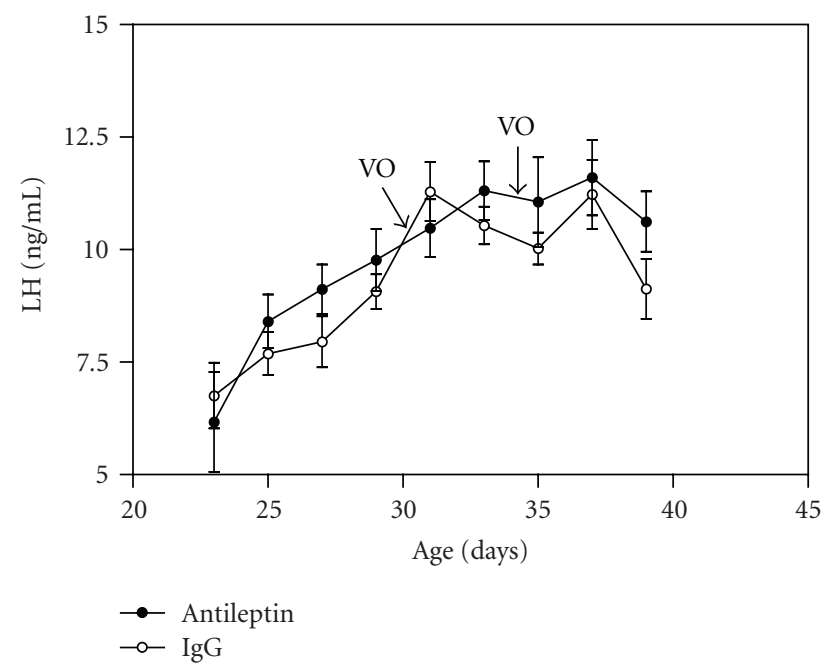

Figure 3: Serum LH concentrations as rats approach and during puberty ( $n=10$ in antileptin group A and $n=11$ in control group $B)$. Data is presented as mean $\pm \operatorname{SEM}(P>.05$ for all ages $)$. Arrows indicate the mean day of $\mathrm{VO}$ (see Figure 1 legend for details).

immunoneutralization of leptin, as in our experiments, had no significant change in circulating leptin compared to $\operatorname{IgG}$ ICV-treated controls [20].

In our studies, at day 35 , day 37 , and day 39 , there were statistically significant increases compared to day 23 in the serum leptin concentrations in group A rats. Seemingly, serum leptin rebounded in antileptin group A after day 35 , however, this was not observed in the control group B 
rats. Tellingly, the body weight on the day of VO in the antileptin treated group was significantly higher compared with that of control group, implying that central leptin is more relevant than body weight in initiating puberty. Also, administration of leptin to female mice (intraperitoneal) or rat (ICV) induced early onset puberty despite attenuated weight gain $[14,23]$.

Peripheral leptin treatment produces central modulation of GnRH secretion and alters LH pulse frequency in food restricted animals [16]. For leptin to be actively involved in initiating puberty, it should act centrally to modify LH release. Serum levels of $\mathrm{LH}$ can serve as an indirect measure of hypothalamic function [11]. Nevertheless, in our rats, serum leptin did not significantly correlate with serum LH. Hiney et al. reported that leptin replacement was able to reverse the suppressed levels of LH caused by ethanol, but the onset of puberty was still delayed [24]. These results buttress our findings that LH is not the sole hormone which initiates puberty $[25,26]$.

The lack of correlation between serum leptin and LH (using our assay methods) suggests that circulating leptin per se does not affect gonadotropin secretion. However, leptin given intraperitoneal reportedly prevents the reduction in LH pulse frequency induced by fasting [16]. Nonetheless, central leptin is a likely modifier of the gonadotropin axis [27]. This supports our finding that there was no difference in circulating leptin or LH levels by our assay method between the two groups.

Almost certainly, VO is determined by ovarian estrogen production. Because serum leptin and $\mathrm{LH}$ were not different between the two groups, yet VO was affected by central antileptin, this raises the possibility of other factors alter gonadal hormone production.

One possibility is that leptin oversees GnRH secretion by acting transynaptically [10]. Yet another mechanistic scenario is that leptin serves to regulate an unknown intermediate which in turn control GnRH [28]. The molecular mechanism and site of action, whereby central leptin governs the onset of puberty of female rat remain unresolved.

We conclude that central leptin, in contrast to that in the periphery, plays a key role in female rat puberty and more so than body mass or serum LH. Finally, central leptin, not serum leptin, may be more germane than body weight on impacting female rat puberty.

\section{Abbreviations}

IVC: Intra-cerebroventricular

IgG: Immunoglobulin G

VO: Vaginal opening

BMI: Body mass index

FSH: Follicle-stimulating hormone

LH: Luteinizing hormone

GnRH: Gonadotropin-releasing hormone

\section{Acknowledgments}

The authors would like to thank Xudong Wang (Department of Pediatric Endocrinology, University of Alabama at Birmingham), Zhijian $\mathrm{Hu}$ (Fujian Medical University) for statistical consultation Professor Donghui Su (Fujian Medical University) for assay consultation, Xiang Jianjun for assisting rat experiments (Fujian Medical University), and Jinglu Zhu and Jinzhu Huang (Fuzhou Children's Hospital of Fujian) for blood sampling. This work was supported by sciences and Technique program of Fujian Province (2002Y049).

\section{References}

[1] Z. M. Van der Spuy, "Nutrition and reproduction," Clinics in Obstetrics and Gynaecology, vol. 12, no. 3, pp. 579-604, 1985.

[2] R. E. Frisch and R. Revelle, "Height and weight at menarche and a hypothesis of menarche," Archives of Disease in Childhood, vol. 46, no. 249, pp. 695-701, 1971.

[3] F. H. Bronson, "Food-restricted, prepubertal, female rats: rapid recovery of luteinizing hormone pulsing with excess food, and full recovery of pubertal development with gonadotropin-releasing hormone," Endocrinology, vol. 118, no. 6, pp. 2483-2487, 1986.

[4] M. J. De Souza and D. A. Metzger, "Reproductive dysfunction in amenorrheic athletes and anorexic patients: a review," Medicine and Science in Sports and Exercise, vol. 23, no. 9, pp. 995-1007, 1991.

[5] J. Argente, I. Barrios, J. A. Chowen, M. K. Sinha, and R. V. Considine, "Leptin plasma levels in healthy Spanish children and adolescents, children with obesity, and adolescents anorexia nervosa and bulimia nervosa," Journal of Pediatrics, vol. 131, no. 6, pp. 833-838, 1997.

[6] Y. Zhang, R. Proenca, M. Maffei, M. Barone, L. Leopold, and J. M. Friedman, "Positional cloning of the mouse obese gene and its human homologue," Nature, vol. 372, no. 6505, pp. 425432, 1994.

[7] I. A. Barash, C. C. Cheung, D. S. Weigle, et al., "Leptin is a metabolic signal to the reproductive system," Endocrinology, vol. 137, no. 7, pp. 3144-3147, 1996.

[8] F. F. Chehab, M. E. Lim, and R. Lu, "Correction of the sterility defect in homozygous obese female mice by treatment with the human recombinant leptin," Nature Genetics, vol. 12, no. 3, pp. 318-320, 1996.

[9] R. S. Ahima, J. Dushay, S. N. Flier, D. Prabakaran, and J. S. Flier, "Leptin accelerates the onset of puberty in normal female mice," The Journal of Clinical Investigation, vol. 99, no. 3, pp. 391-395, 1997.

[10] M. J. Cunningham, D. K. Clifton, and R. A. Steiner, "Leptin's actions on the reproductive axis: perspectives and mechanisms," Biology of Reproduction, vol. 60, no. 2, pp. 216-222, 1999.

[11] W. H. Yu, M. Kimura, A. Walczewska, S. Karanth, and S. M. Mccann, "Role of leptin in hypothalamic-pituitary function," Proceedings of the National Academy of Sciences of the United States of America, vol. 94, no. 3, pp. 1023-1028, 1997.

[12] M. Tezuka, M. Irahara, K. Ogura, et al., "Effects of leptin on gonadotropin secretion in juvenile female rat pituitary cells," European Journal of Endocrinology, vol. 146, no. 2, pp. 261266, 2002.

[13] O. J. Ponzo, B. Szwarcfarb, D. Rondina, et al., "Changes in the sensitivity of gonadotrophi axis to leptin during sexual maturation in female rats," Neuroendocrinology Letters, vol. 22, no. 6 , pp. $427-431,2001$. 
[14] F. F. Chehab, K. Mounzih, R. Lu, and M. E. Lim, "Early onset of reproductive function in normal female mice treated with leptin," Science, vol. 275, no. 5296, pp. 88-90, 1997.

[15] S. J. Nazian and D. F. Cameron, "Temporal relation between leptin and various indices of sexual maturation in the male rat," Journal of Andrology, vol. 20, no. 4, pp. 487-491, 1999.

[16] S. Nagatani, P. Guthikonda, R. C. Thompson, H. Tsukamura, K.-I. Maeda, and D. L. Foster, "Evidence for GnRH regulation by leptin: leptin administration prevents reduced pulsatile $\mathrm{LH}$ secretion during fasting," Neuroendocrinology, vol. 67, no. 6, pp. 370-376, 1998.

[17] R. S. Ahima, et al., "Role of leptin in the neuroendocrine response to fasting," Nature, vol. 382, no. 6588, pp. 250-252, 1996.

[18] A. Strobel, T. Issad, L. Camoin, M. Ozata, and A. D. Strosberg, "A leptin missense mutation associated with hypogonadism and morbid obesity," Nature Genetics, vol. 18, no. 3, pp. 213215, 1998.

[19] K. Clément, C. Vaisse, N. Lahlou, et al., "A mutation in the human leptin receptor gene causes obesity and pituitary dysfunction," Nature, vol. 392, no. 6674, pp. 398-401, 1998.

[20] S. Zeinoaldini, J. J. M. Swarts, and B. J. M. Van de Heijning, "Chronic leptin infusion advances, and immunoneutralization of leptin postpones puberty onset in normally fed and feed restricted female rats," Peptides, vol. 27, no. 7, pp. 16521658, 2006.

[21] H. Watanobe and H. B. Schiöth, "Postnatal profile of plasma leptin concentrations in male and female rats: relation with the maturation of the pituitary-gonadal axis," Regulatory Peptides, vol. 105, no. 1, pp. 23-28, 2002.

[22] T. M. Plant and A. R. Durrant, "Circulating leptin does not appear to provide a signal for triggering the initiation of puberty in the male rhesus monkey (Macaca mulatta)," Endocrinology, vol. 138, no. 10, pp. 4505-4508, 1997.

[23] N. M. Gruaz, M. Lalaoui, D. D. Pierroz, et al., "Chronic administration of leptin into the lateral ventricle induces sexual maturation in severely food-restricted female rats," Journal of Neuroendocrinology, vol. 10, no. 8, pp. 627-633, 1998.

[24] J. K. Hiney, R. K. Dearth, F. Lara III, S. Wood, V. Srivastava, and W. Les Dees, "Effects of ethanol on leptin secretion and the leptin-induced luteinizing hormone ( $\mathrm{LH})$ release from late juvenile female rats," Alcoholism: Clinical and Experimental Research, vol. 23, no. 11, pp. 1785-1792, 1999.

[25] P. D. Finn, M. J. Cunningham, K.-Y. F. Pau, H. G. Spies, D. K. Clifton, and R. A. Steiner, "The stimulatory effect of leptin on the neuroendocrine reproductive axis of the monkey," Endocrinology, vol. 139, no. 11, pp. 4652-4662, 1998.

[26] M.-L. Hakansson, H. Brown, N. Ghilardi, R. C. Skoda, and B. Meister, "Leptin receptor immunoreactivity in chemically defined target neurons of the hypothalamus," Journal of Neuroscience, vol. 18, no. 1, pp. 559-572, 1998.

[27] A. R. Maqsood, J. A. Trueman, A. J. Whatmore, et al., "The relationship between nocturnal urinary leptin and gonadotrophins as children progress towards puberty," Hormone Research, vol. 68, no. 5, pp. 225-230, 2007.

[28] R. Reynoso, O. J. Ponzo, B. Szwarcfarb, et al., "Effect of leptin on hypothalamic release of gnrh and neurotransmitter amino acids during sexual maturation in female rats," Experimental and Clinical Endocrinology and Diabetes, vol. 111, no. 5, pp. 274-277, 2003. 